\title{
Institutional implementation of clinical tumor profiling on an unselected cancer population
}

\author{
Lynette M. Sholl, ${ }^{1}$ Khanh Do, ${ }^{2,3}$ Priyanka Shivdasani, ${ }^{1}$ Ethan Cerami, ${ }^{4}$ Adrian M. Dubuc, ${ }^{1}$
} Frank C. Kuo, ${ }^{1}$ Elizabeth P. Garcia, ${ }^{1}$ Yonghui Jia, ${ }^{1}$ Phani Davineni, ${ }^{1}$ Ryan P. Abo, ${ }^{1,5}$ Trevor J. Pugh, ${ }^{6}$ Paul van Hummelen, ${ }^{4}$ Aaron R. Thorner, ${ }^{5}$ Matthew Ducar, ${ }^{1,5}$ Alice H. Berger,,${ }^{2,7}$ Mizuki Nishino, ${ }^{8}$ Katherine A. Janeway, ${ }^{9}$ Alanna Church, ${ }^{10}$ Marian Harris, ${ }^{10}$ Lauren L. Ritterhouse, ${ }^{1}$ Joshua D. Campbell, ${ }^{2}$ Vanesa Rojas-Rudilla, ${ }^{1,2}$ Azra H. Ligon, ${ }^{1}$ Shakti Ramkissoon, ${ }^{1}$ James M. Cleary, ${ }^{2,3}$ Ursula Matulonis, ${ }^{2}$ Geoffrey R. Oxnard, ${ }^{2}$ Richard Chao, ${ }^{11}$ Vanessa Tassell, ${ }^{11}$ James Christensen, ${ }^{11}$ William C. Hahn, ${ }^{2,5,7,12}$ Philip W. Kantoff,, ${ }^{12}$ David J. Kwiatkowski, ${ }^{2}$ Bruce E. Johnson, ${ }^{2}$ Matthew Meyerson, ${ }^{1,2,5,7}$ Levi A. Garraway, ${ }^{2,7,13}$ Geoffrey I. Shapiro, ${ }^{2,3}$ Barrett J. Rollins, ${ }^{2}$ Neal I. Lindeman, ${ }^{1}$ and Laura E. MacConaill ${ }^{1,5}$

'Department of Pathology, Brigham and Women's Hospital, Boston, Massachusetts, USA. 'Department of Medical Oncology, Dana-Farber Cancer Institute (DFCI), Boston, Massachusetts, USA; Department of Medicine, Brigham and Women's Hospital, Boston, Massachusetts, USA. ${ }^{3}$ Early Drug Discovery Center, ${ }^{4}$ Department of Biostatistics and Computational Biology, and ${ }^{5}$ Center for Cancer Cenome Discovery, DFCI, Boston, Massachusetts, USA. ${ }^{6}$ Princess Margaret Cancer Centre, University Health Network, Toronto, Ontario, Canada; Department of Medical Biophysics, University of Toronto, Ontario, Canada. ${ }^{7}$ Broad Institute of Harvard and MIT, Cambridge, Massachusetts, USA. ${ }^{8}$ Department of Radiology, DFCl and Brigham and Women's Hospital, Boston, Massachusetts, USA. 'Dana-Farber/Boston Children's Cancer and Blood Disorders Center, Boston, Massachusetts, USA. ${ }^{10}$ Department of Pathology, Boston Children's Hospital, Boston, Massachusetts, USA. "'Mirati Therapeutics, San Diego, California. ${ }^{12}$ Lank Center for Genitourinary Oncology and ${ }^{13}$ Center for Cancer Precision Medicine, DFCI, Boston, Massachusetts, USA.

Conflict of interest: M. Nishino is a consultant for Bristol-Myers Squibb. G.R. Oxnard is a consultant for AstraZeneca, Ariad, and Boehringer-Ingelheim and has honoraria from AstraZeneca and Chugai. L.M. Sholl is a consultant for Cenentech. M. Meyerson has research funding from Bayer and a patent on the use of ECFR mutation analysis for lung cancer diagnosis and is a founder of Foundation Medicine. L.A. Garraway is a founder, consultant, and equity holder in Foundation Medicine.

Submitted: February 17, 2016 Accepted: October 13, 2016 Published: November 17, 2016

Reference information:

JCI Insight. 2016;1(19):e87062. doi:10.1172/jici.insight.87062.
BACKGROUND. Comprehensive genomic profiling of a patient's cancer can be used to diagnose, monitor, and recommend treatment. Clinical implementation of tumor profiling in an enterprisewide, unselected cancer patient population has yet to be reported.

METHODS. We deployed a hybrid-capture and massively parallel sequencing assay (OncoPanel) for all adult and pediatric patients at our combined cancer centers. Results were categorized by pathologists based on actionability. We report the results for the first 3,727 patients tested.

RESULTS. Our cohort consists of cancer patients unrestricted by disease site or stage. Across all consented patients, half had sufficient and available ( $>20 \%$ tumor) material for profiling; once specimens were received in the laboratory for pathology review, $73 \%$ were scored as adequate for genomic testing. When sufficient DNA was obtained, OncoPanel yielded a result in $96 \%$ of cases. $73 \%$ of patients harbored an actionable or informative alteration; only $19 \%$ of these represented a current standard of care for therapeutic stratification. The findings recapitulate those of previous studies of common cancers but also identify alterations, including in AXL and ECFR, associated with response to targeted therapies. In rare cancers, potentially actionable alterations suggest the utility of a "cancer-agnostic" approach in genomic profiling. Retrospective analyses uncovered contextual genomic features that may inform therapeutic response and examples where diagnoses revised by genomic profiling markedly changed clinical management.

CONCLUSIONS. Broad sequencing-based testing deployed across an unselected cancer cohort is feasible. Genomic results may alter management in diverse scenarios; however, additional barriers must be overcome to enable precision cancer medicine on a large scale.

FUNDING. This work was supported by DFCl, BWH, and the National Cancer Institute (5R33CA155554 and 5K23CA157631). 


\section{Introduction}

In cancer medicine, knowledge of specific tumor genomic alterations has become increasingly important in diagnosis, prognosis, and treatment. The clinical benefits of identification and targeted inhibition of driver genomic alterations have been demonstrated in multiple cancer types (1-7). These successes have led to the notion that systematic assessment of "actionable" cancer genomic changes may enable precision oncology $(8,9)$.

To identify candidates for both FDA-approved targeted therapies and for enrollment into molecularly driven clinical trials, molecular diagnostics laboratories have historically provided a menu of single-target assays. However, sequential testing of multiple genes can rapidly exhaust precious tumor material, and the testing increases complexity, cost, and turnaround time. Massively parallel sequencing (MPS) offers several advantages over the single-gene approach (10-15); most importantly, it offers a more sensitive and more comprehensive genomic profile that does not apply an a priori knowledge of alterations that may be more common in a specific tumor type. Several academic centers have demonstrated that large MPS panels can be employed in practice $(11,16,17)$, with a number of groups reporting a high rate of "actionable" (informative or clinically relevant) gene alterations in clinical cancer cohorts $(4,18,19)$.

While the high proportions of actionable alterations in cancer cohorts seems encouraging, the clinical utilization of broad genomic profiling is nascent and the eventual clinical utility has yet to be determined. Several recent reports have shown that only a small proportion of patients $(\sim 5 \%-10 \%)$ have their cancer genomic data used as a criterion for selection of a targeted therapy or a clinical trial $(16,19,20)$. A variety of logistic, operational, and medical reasons (20) have been cited, such as identification of the appropriate genomic target at the right time in a patient's clinical course, time to result, the value of biopsy at the time of progressive disease (21), choice of conventional treatment protocols, access to clinical trials $(16,20)$ or off-label use of drug (19), and patient preferences (20). Similarly, evaluation of the confidence of physicians at our institutions in using genomic data to make decisions on patient treatment options (22) indicates that not all "actionable" genomic data will be acted upon.

Assessment of the clinical utility (i.e., measuring outcomes) of applying molecularly targeted therapies has delivered generally encouraging, though mixed, results $(4,18,19,21,23-25)$, likely based in part on the maturity of targeted therapies in different tumor types and small and heterogenous patient populations, often with metastatic disease and multiple prior therapies. To date, the potential for enterprise-wide clinical tumor profiling to alter diagnosis or guide the clinical management of patients remains incompletely ascertained.

Starting in 2011, we have offered genomic profiling to all cancer patients seen at DFCI, Brigham and Women's Hospital, and Boston Children's Hospital. Through this initiative - termed Profile - we have analyzed over 15,000 individual tumors to date, including 5,000 by genotyping (OncoMap) (26) and >12,000 by MPS (OncoPanel), of which the first approximately 3,700 cases are reported here. The Profile initiative captures the entire population of cancer patients seen at our institutes, thereby defining the plausibly actionable pan-cancer genome in clinical practice. We reasoned that a systematic analysis of the clinical, pathologic, and genomic context of tumor mutations from this study might offer diagnostic clarification and identify new genomic predictors of response to targeted therapy in a manner that enables future large-scale cancer precision medicine initiatives, while, at the same time, identifying challenges and barriers to widespread implementation of precision medicine that would inform subsequent phases of our initiative.

\section{Results}

Overall program and technical performance of OncoPanel. Our programmatic approach is summarized in Figure 1A. Our overall consent rate was approximately $70 \%$. Tumor material was available for review in $72 \%$ and was adequate for sequencing in 53\% of consented patients (Figure 1B). Of patients for whom material was in hand, $23 \%$ did not have sufficient material for testing for a variety of reasons, including a lack of an invasive cancer diagnosis (e.g., squamous cell cancer in situ, breast ductal carcinoma in situ), inappropriate fixation (decalcification), protocol-excluded specimens (breast core biopsies), or specimens yielding less than 50 ng DNA. A small proportion of pathologically adequate specimens (4\%) failed sequencing. 3,892 samples were tested during the first year; cancer types are listed in Supplemental Table 2 (supplemental material available online with this article; doi:10.1172/jci.insight.87062DS1). Once sufficient DNA was obtained, sequencing was successful in 3,727 cases (96\% success rate; Table 1). Sequencing failure rates were significantly higher for consult cases $(7 \%)$ as compared with in-house cases $(3 \%)(P<0.001)$ (Figure 1B). The success rate in specimens with adequate tumor cells ranged from a low of $91 \%$ in breast tumors to 
Enterprise-level testing

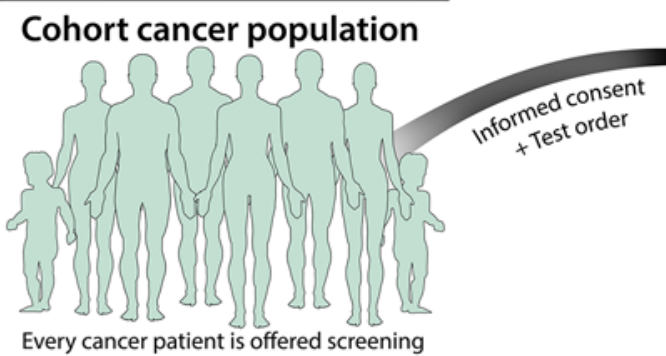
Genomic test performed
on tumor specimen Analysis and interpretation of data
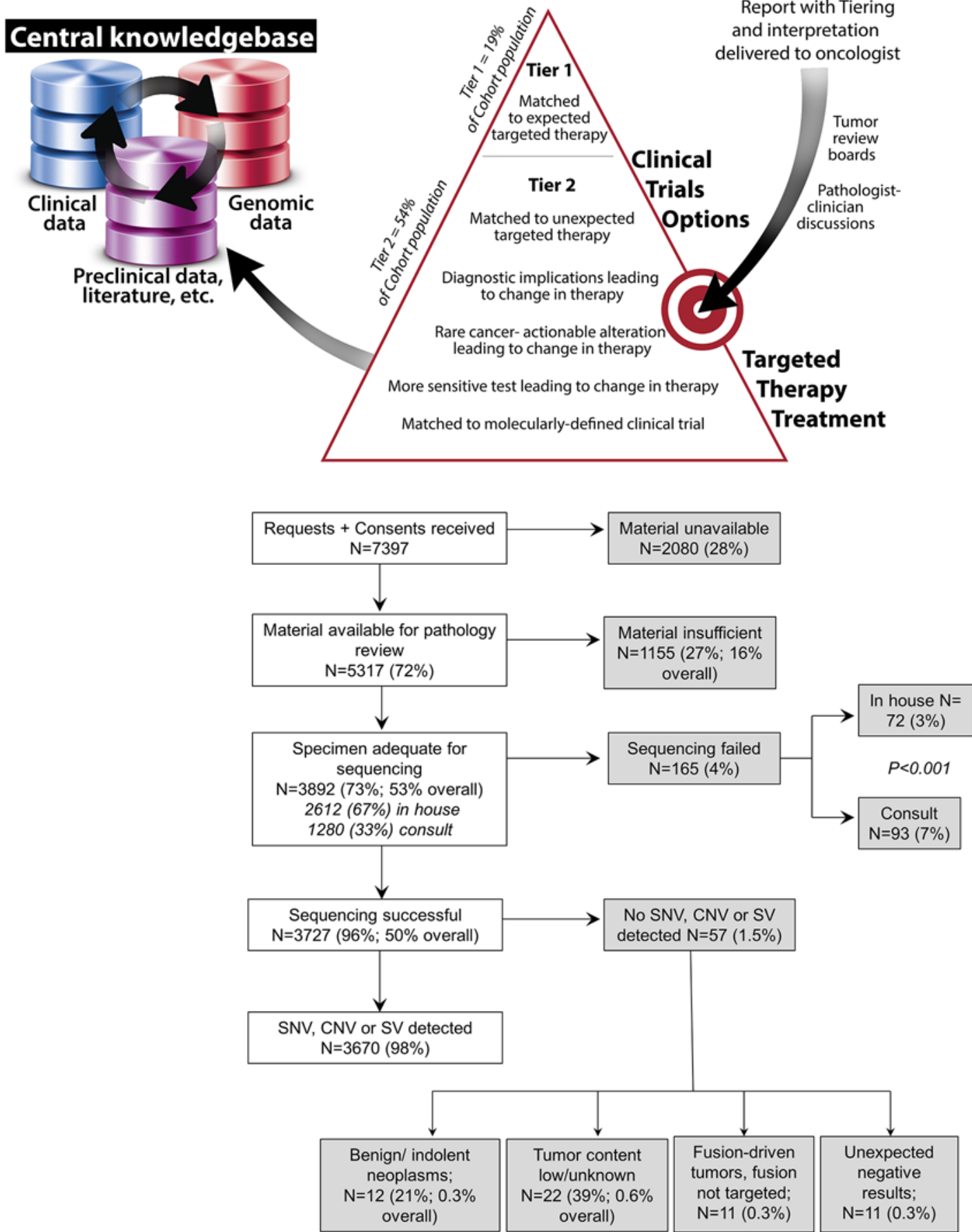
Figure 1. An overview of the Profile program, including specimen availability, adequacy, and sequencing success. (A) For consented patients, a cancer specimen is genomically profiled in a CLIA laboratory. Results are tiered by a team that interprets pathology, incorporating information from each patient's electronic health record and provided to the patient's treating physician(s). Genomic, pathologic, and clinical data are deposited in a central knowledge base that can link to full clinical annotation. The knowledge base can be queried to facilitate development and enrollment of basket trials and inform tumor board discussions. (B) Once patient consent and a test requisition are received in the laboratory, pathology records are reviewed for available cancer specimens. Unavailable materials are defined as those not physically located within the participating institutions and not actively requested by the treating physician for OncoPanel testing. Cases are most commonly insufficient because material is too small, tumor content is less than $20 \%$, or generates $<50 \mathrm{ng}$ of DNA. Sequencing failure is defined as mean target coverage of less than 50 reads. Tumors lacking single nucleotide variation (SNV), copy number variation (CNV), or structural variants (SV) were rare, but 22 were cases with low tumor content. Reported $P$ value calculated by Fisher's exact test.

$>99 \%$ in hematologic and pancreaticobiliary/liver tumors. The reasons for the substantially higher failure rate in breast tumors are not entirely clear; one potential explanation is that for much of the testing period breast core needle biopsies were excluded from testing in order to preserve tissue for clinical FISH and expression profiling assays. Therefore, most eligible specimens were either mastectomies/lumpectomies or metastatic biopsies. These specimens may be prone to underfixation or harsh fixation, contributing to DNA degradation. The average turnaround time for the test - from receipt of sample in the lab, through plate assembly and chemistries, sequencing, technical review, interpretation, and reporting - was 5.3 weeks.

In addition to evaluating clinical performance in the technical validation (see Methods), we calculated sensitivity and specificity for each category of genomic alteration using hallmark examples that had orthogonal, consecutive clinical testing and known "true positive" specimens. OncoPanel was $100 \%$ sensitive (95\% CI $0.93-1)$ and $100 \%$ specific (95\% CI $0.96-1)$ for KRAS exon 12-13 mutation detection as compared with pyrosequencing. Isolated cases of false-positive, low-level single nucleotide mutation events were detected but were generally attributable to cross contamination rather than sequencing error, an issue that was subsequently addressed by implementation of parallel "fingerprinting," using a genotyping assay on an aliquot of sample DNA (see Methods). Some insertion-deletion events are not readily detected using MPS-based techniques due to informatics challenges relating to sequence alignment (27). We therefore utilized multiple informatics tools (GATK and Breakmer), as well as forced manual review, for selected clinically important variant hot spots, in order to maximize our ability to detect small- to medium-sized insertion-deletion mutations. The forced review process allowed manual recovery of $12.5 \%$ of indels in NPM1 and $100 \%$ of indels in FLT3 (our algorithmic approaches were unable to detect FLT3 internal tandem duplications). OncoPanel was 100\% sensitive (95\%CI $0.8-1)$ and $100 \%$ specific (95\% CI 0.98-1) for EGFR exon 19 deletion mutation detection as compared with PCR-based sizing assays and/or Sanger sequencing with peptide nucleic acid clamps. OncoPanel was similarly $100 \%$ sensitive (95\% CI $0.85-1)$ and $100 \%$ specific (95\% CI $0.92-1)$ for EGFR high-level amplification in glioblastoma. The performance of OncoPanel for detection of single-copy, chromosomal arm-level events was also robust but less specific (97.5\% sensitivity [95\% CI 0.85-0.99] and $83.3 \%$ specificity [95\% CI $0.51-0.97$ ]) for detection of the diagnostic $1 \mathrm{p} / 19 \mathrm{q}$ deletion event in oligoastrocytomas or oligodendrogliomas, as compared with high resolution aCGH or FISH (28) (Supplemental Table 3 and 4). Low-level copy number calling in particular was adversely affected by sample DNA degradation or low tumor content. Of 190 lung adenocarcinomas tested for ALK fusions by FISH or immunohistochemistry, OncoPanel was $94 \%$ sensitive (95\% CI 0.69-0.99) and 100\% specific (95\% CI 0.98-1) (Supplemental Table 5). Moreover, OncoPanel detected an $A L K$ fusion in one case with a reported FISH-negative result; the patient was switched to crizotinib therapy and experienced disease stabilization.

Pan-cancer overview of results from the cohort. Overall, the recurrent gene alterations were observed at the expected frequencies (Figure 2). To analyze these genes from the perspective of actionability, we categorized the alterations into tiers (Table 2). $73 \%$ of the cohort showed at least one "clinically actionable or informative" alteration (tier 1 or 2; Figure 1A). 19\% of alterations would inform standard-of-care therapeutic decision-making based on diagnostic, prognostic, or predictive impact and were grouped into tier 1. $54 \%$ of alterations may be used as the basis for recommending enrollment on trials of approved or investigational agents or may be used for informing diagnosis; these were grouped into tier 2 (Figure 1A). Tier 3 alterations were considered to be biologically relevant but were unlikely to inform current treatment decisions. Variants of unknown significance were categorized as tier 4. Known germline polymorphisms that were not filtered by the informatics pipeline were categorized as tier 5 . 
Table 1. Assay success and mutation rates across disease sites

\begin{tabular}{lcccc}
\hline Disease site & Number tested & Successful cases (\%) & Average mutation count (per Mb & Mutation count range \\
Breast & 291 & $257(88)$ & $7.3(5.4)$ & $0-55$ \\
CNS & 432 & $404(94)$ & $7.7(5.7)$ & $0-209$ \\
Endocrine & 122 & $118(97)$ & $4.3(3.2)$ & $0-34$ \\
Gastrointestinal & 314 & $304(97)$ & $10.7(7.9)$ & $0-155$ \\
Gynecology Tract & 590 & $553(94)$ & $11.1(8.2)$ & $0-227$ \\
Head and neck & 169 & $160(95)$ & $5(3.6)$ & $0-410$ \\
Heme malignancies & 339 & $336(99)$ & $4.3(3.2)$ & $0-40$ \\
Kidney & 105 & $105(100)$ & $5.5(4)$ & $0-17$ \\
Pancreaticobiliary and liver & 89 & $87(98)$ & $7.6(5.6)$ & $0-44$ \\
Pediatric & 93 & $93(100)$ & $3.5(2.6)$ & $0-174$ \\
Prostate & 184 & $170(92)$ & $5.6(4.1)$ & $0-41$ \\
Sarcoma & 181 & $178(98)$ & $17.6(13)$ & $0-36$ \\
Skin & 70 & $66(94)$ & $4.5(3.3)$ & $0-134$ \\
Testis & 42 & $39(93)$ & $8.9(6.6)$ & $0-23$ \\
Thoracic & 732 & $723(99)$ & $17.5(12.9)$ & $0-70$ \\
Unknown & 12 & $12(100)$ & $11.8(8.7)$ & $2-49$ \\
Urinary Tract & 127 & $122(96)$ & $8.6(6.3)$ & $1-82$ \\
Total & 3,892 & $3,727(96)$ & $0-410$ \\
AThis assay sequences 1.358235 megabases $(M b)$ & & & \\
\hline
\end{tabular}

The top tier variants for all cases, excluding those cases with only tier 4 variants, are included in Supplemental Table 6. The most common tier 1 alterations were ERBB2 amplification; EGFR L858R, $B R A F$ V600E in melanoma and colon adenocarcinoma and KRAS codon 12 and 13 mutations in colon and lung adenocarcinomas; EGFR exon 19 deletions; and $A L K$ and $A B L 1$ rearrangements. The most frequently observed tier 2 alterations were amplifications of $C D K 4, C C N D 1$, and CCNE1; homozygous deletion of $C D K N 2 A$; and single nucleotide variation of $I D H 1 \mathrm{R} 132 \mathrm{H}$ and $B R A F$ V600E in lung adenocarcinoma and other tumor types and KRAS codon 12 outside of the setting of colon and lung cancers. The most common tier 3 alterations were amplification of $M D M 2$ and single nucleotide variations in $A P C, A S X L 1, A T M, I D H 1, T E T 2$, and TP53. In contrast to previous large-scale genomic studies of individual tumor types, most of which are biased toward analysis of primary tumors, this

Table 2. Classification schema according to variant actionability

\begin{tabular}{|c|c|c|}
\hline Tier & Explanation & $\begin{array}{l}\text { No. of cases with at least } \\
\text { one variant in the tier (\%) }\end{array}$ \\
\hline
\end{tabular}

1 Published evidence confirming clinical utility in the assigned tumor type. This utility may include the following:

(a) Predicting response to an FDA-approved therapy

(b) Establishing prognosis in a manner that affects therapeutic decision making

(c) Conferring risk of inherited cancer syndrome.

$714(19)$

2

(a) Established biomarker for clinical trial eligibility

(b) Limited evidence of prognostic association

(c) Predictive of response to FDA-approved therapy in another tumor type

(d) Similar to another mutation proven to be predictive of response to FDA-approved therapy in this tumor type

$2,001(54)$

3 Uncertain clinical utility but some evidence of biologic relevance in the form of the following

(a) Preclinical studies demonstrating association with response to therapy in this tumor type

(b) Alteration in a highly conserved region of the protein predicted to alter function

(c) Selection of investigational therapy in another tumor type

470 (13)

$4 \quad$ Novel variant of unknown significance in cancer

473 (13)

Total

Not reported

3,658 (98)

ESP, Exome Sequencing Project. 


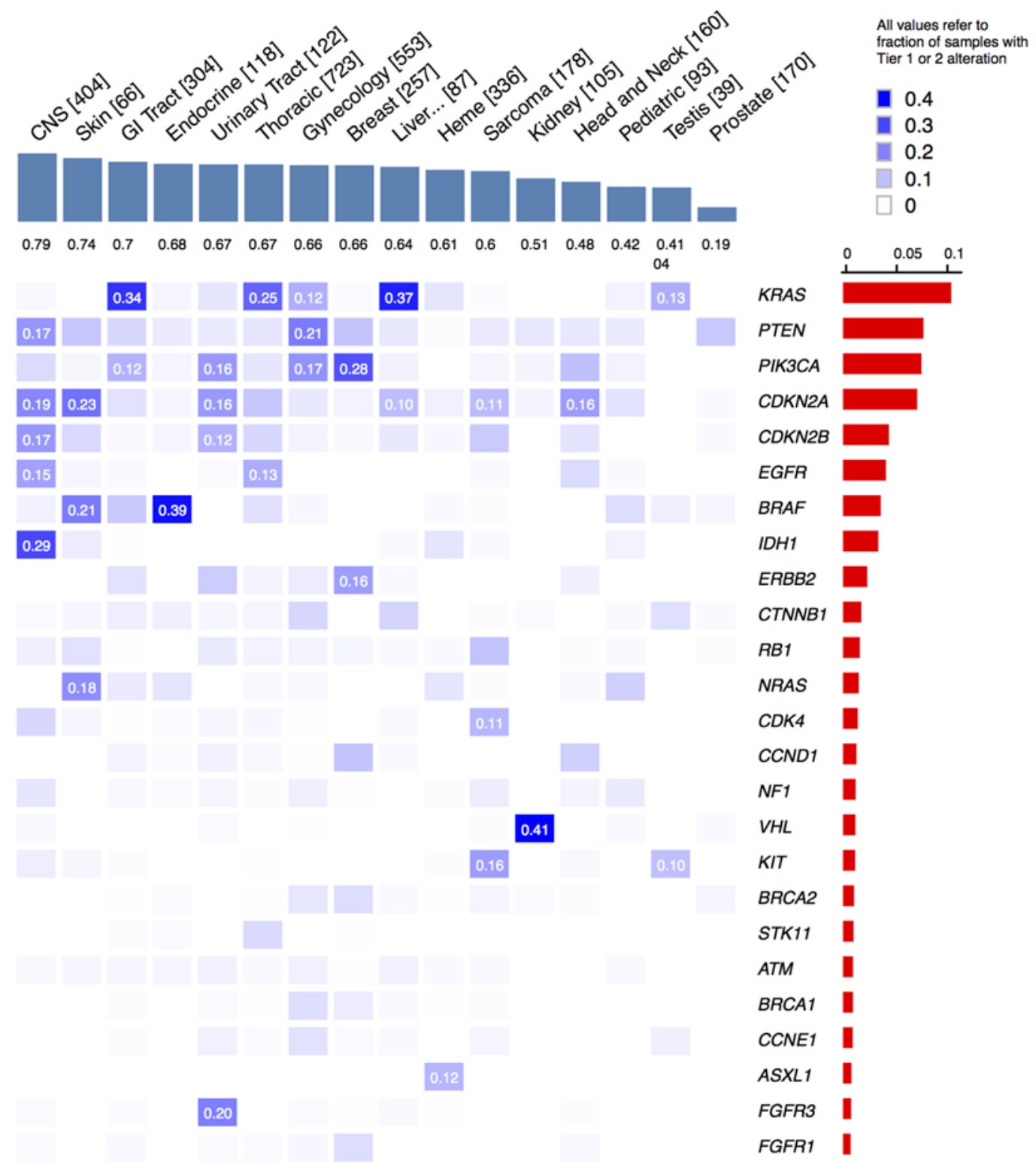

Figure 2. Frequency of alterations across the Profile cohort. The 25 most commonly altered actionable genes across all disease sites (with implications for FDA-approved targeted therapies or clinical trial enrollment) are shown. The frequency of tier 1 and 2 actionable alterations ranged from $79 \%$ of primary CNS tumors to $19 \%$ of prostate adenocarcinomas.

initiative examined $734(20 \%)$ specimens taken from metastatic sites. As a result, we can begin to discern distinct patterns between primary and metastatic tumors across the cancer genome and within tumor types on the population level. In lung adenocarcinoma, alterations that were significantly more common in metastases as compared with primaries included TP53 mutations (128 of 281 primaries, 115 of 193 metastases; $P=0.007$ ) and $C D K N 2 A$ mutations or homozygous deletions (18 of 281 primaries, 29 of 193 metastases; $P=0.018$ ). In colon carcinoma, TP53 mutations and MYC amplification were significantly enriched in metastases (TP53: 99 of 144 primaries, 43 of 48 metastases; $P=0.045$ and MYC: 6 of 144 primaries, 8 of 48 metastases; $P=0.036$, respectively). (Supplemental Figure 1). Because variable tumor purity may affect the rates of variant detection, particularly for copy number alterations, we also compared the histologic estimates of tumor content. Although lung adenocarcinoma metastases had a statistically significantly higher average tumor purity as compared with primaries (53\% versus $48 \%, P=0.01$ ), this difference is unlikely to be clinically significant. The average tumor purity was similar between colon metastases and primaries $(54 \%$ versus $53 \%, P=0.57)$. 
Table 3. OncoPanel sequencing leading to a change in diagnosis

\begin{tabular}{|c|c|c|c|c|c|}
\hline Case & Original diagnosis & Patient characteristics & OncoPanel findings & New diagnosis & Clinical implications \\
\hline 1 & $\begin{array}{l}\text { Peripheral T cell lymphoma, } \\
\text { revised to myeloid sarcoma }\end{array}$ & $\begin{array}{c}35 \text { male, refractory to } 5 \text { lines of } \\
\text { therapy }\end{array}$ & FIP1L1-PDGFRA fusion & $\begin{array}{l}\text { FIP1L1-PDGFRA-driven } \\
\text { AML (confirmed by } \\
\text { FISH) }\end{array}$ & $\begin{array}{l}\text { Patient initiated on } \\
\text { imatinib therapy with } \\
\text { immediate response. } \\
\text { Allogeneic SCT with } \\
\text { NED at } 1 \text { year. }\end{array}$ \\
\hline 2 & $\begin{array}{l}\text { Small-cell carcinoma, revised to } \\
\text { atypical carcinoid }\end{array}$ & $\begin{array}{l}\text { 42-year-old man, light smoker, } \\
\text { PD on cisplatin-etoposide }\end{array}$ & EWSR1-ERG fusion & $\begin{array}{l}\text { Ewing's sarcoma } \\
\text { (confirmed by FISH) }\end{array}$ & $\begin{array}{c}\text { Patient stable on } \\
\text { palliative topotecan } \\
\text { and cytoxan at 2-year } \\
\text { follow-up. }\end{array}$ \\
\hline 3 & Uterine leiomyosarcoma & $\begin{array}{l}\text { 53-year-old woman with } \\
\text { "STUMP" treated by } \\
\text { morcellation; recurred as } \\
\text { peritoneal dissemination }\end{array}$ & IGFBP5-ALK & $\begin{array}{l}\text { Inflammatory } \\
\text { myofibroblastic tumor } \\
\text { (confirmed by FISH) }\end{array}$ & ALK inhibitor therapy \\
\hline 4 & $\begin{array}{l}\text { Undifferentiated sarcoma, high } \\
\text { grade }\end{array}$ & $\begin{array}{l}\text { 48-year-old woman with rectal } \\
\text { bleeding, 6-cm ileal mass }\end{array}$ & $\begin{array}{l}\text { KIT exon } 11 \text { deletion } \\
\text { mutation }\end{array}$ & $\begin{array}{l}\text { Gastrointestinal } \\
\text { stromal sarcoma (IHC } \\
\text { confirmed KIT and } \\
\text { DOG-1 expression) }\end{array}$ & $\begin{array}{l}\text { Patient initiated on } \\
\text { imatinib therapy with } \\
\text { NED at } 6 \text { months }\end{array}$ \\
\hline 6 & $\begin{array}{l}\text { Undifferentiated sarcoma vs. } \\
\text { carcinoma }\end{array}$ & $\begin{array}{l}\text { 49-year-old woman with } \\
\text { large tumor masses involving } \\
\text { bilateral fallopian tubes, } \\
\text { ovaries, uterus, and sigmoid } \\
\text { colon }\end{array}$ & $\begin{array}{l}\text { MMRd mutation } \\
\text { signature }\end{array}$ & $\begin{array}{l}\text { Undifferentiated } \\
\text { endometrial } \\
\text { adenocarcinoma }\end{array}$ & $\begin{array}{l}\text { Patient DOD; however, } \\
\text { follow-up testing } \\
\text { advised to determine } \\
\text { if sporadic or germline } \\
\text { MMR defect for family } \\
\text { counseling }\end{array}$ \\
\hline 7 & Lung squamous cell carcinoma & $\begin{array}{l}56 \text {-year-old man with multiple } \\
\text { right middle lobe lung masses }\end{array}$ & UVA mutation signature & $\begin{array}{l}\text { Metastatic cutaneous } \\
\text { squamous cell } \\
\text { carcinoma }\end{array}$ & $\begin{array}{l}\text { Triggered chart review: } \\
\text { patient with remote } \\
\text { history of widely } \\
\text { invasive cutaneous } \\
\text { basosquamous } \\
\text { carcinoma }\end{array}$ \\
\hline
\end{tabular}

AML, acute myelogenous leukemia; DOD, dead of disease; GIST, gastrointestinal stromal tumor; IHC, immunohistochemistry; MMRd, mismatch repair deficiency; NED, no evidence of disease; PD, progressive disease; SCT, stem cell transplant; STUMP, smooth muscle tumor of unknown malignant potential

Fifty-seven samples ( $1.5 \%$ overall) had no detectable alterations. Of these, 22 were estimated to have $20 \%$ tumor or less on additional review. These data correlate with our limit of detection experiments. The remaining cases with no detectable alterations may be genomically silent or driven by epigenetic or other alterations (including fusions) not captured by this panel.

Diagnostic revisions enabled by tumor mutation profiling. Despite having undergone expert pathology review in a high volume tertiary center, where our rate of major diagnostic revisions based on histopathologic and immunohistochemical review is about $4 \%$, sequencing results provided clarification or revision of the diagnosis in a number of cases, often with significant implications for clinical management and prognosis. Exemplary cases are listed in Table 3, highlighting the potential utility of sequencing in tumors with unclear pathologic diagnoses. In case 1, a patient with extensive lymphadenopathy and marrow infiltration received various diagnoses at different institutions, including peripheral $\mathrm{T}$ cell lymphoma and myeloid sarcoma. Unexpectedly, OncoPanel data led to a revised diagnosis of FIP1L1-PDGFRA-driven AML. Consequently, imatinib therapy was administered, and a dramatic and sustained clinical response ensued (29). The identification of relatively specific driver alterations was particularly informative in the context of sarcoma, as might be expected based on the frequency of characteristic fusion and mutational alterations in subsets of these tumors. In case 2, a small round blue cell tumor in a nonsmoking man was originally classified as small-cell carcinoma and then as atypical carcinoid tumor; this diagnosis was revised to Ewing's 
A

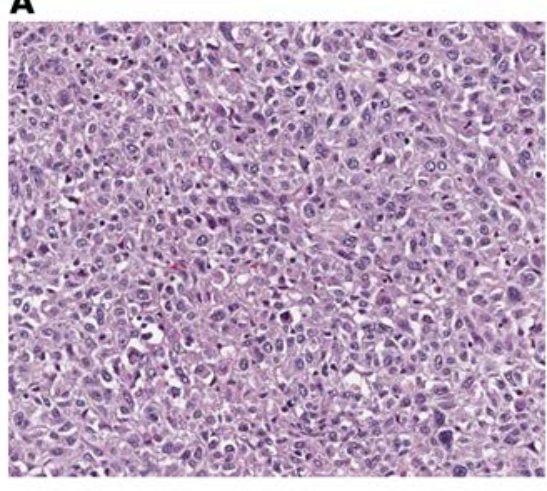

B

TSC2 c. 1249 C $>$ T (p.Q417*) in $84 \%$ of 13 reads TP53 c.166_167GA>A (p.E56fs) in $62 \%$ of 29 reads

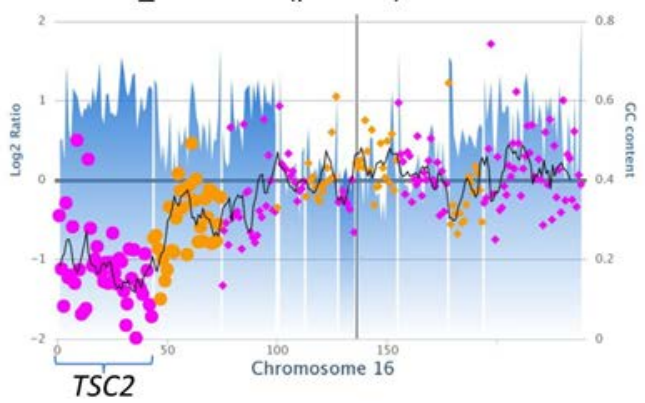

Figure 3. Targeting TSC2 loss of function.

(A) A woman with high-grade serous ovarian carcinoma (original magnification, $\times 200$ ) has evidence for (B) biallelic TSC2 loss, including a truncating mutation and loss of one copy of the gene, based on OncoPanel sequencing. Magenta and gold dots indicate log2 ratio of sample copy number relative to a pooled normal at the level of individual exons and selected introns for each of the targeted genes. Pale blue tracing shows the percent guanine and cytosine (GC) content in the targeted region. (C) Following therapy with everolimus, the woman's CA-125 levels dropped markedly following one cycle of therapy, and CA-125 levels remained low beyond the third cycle of therapy.
C

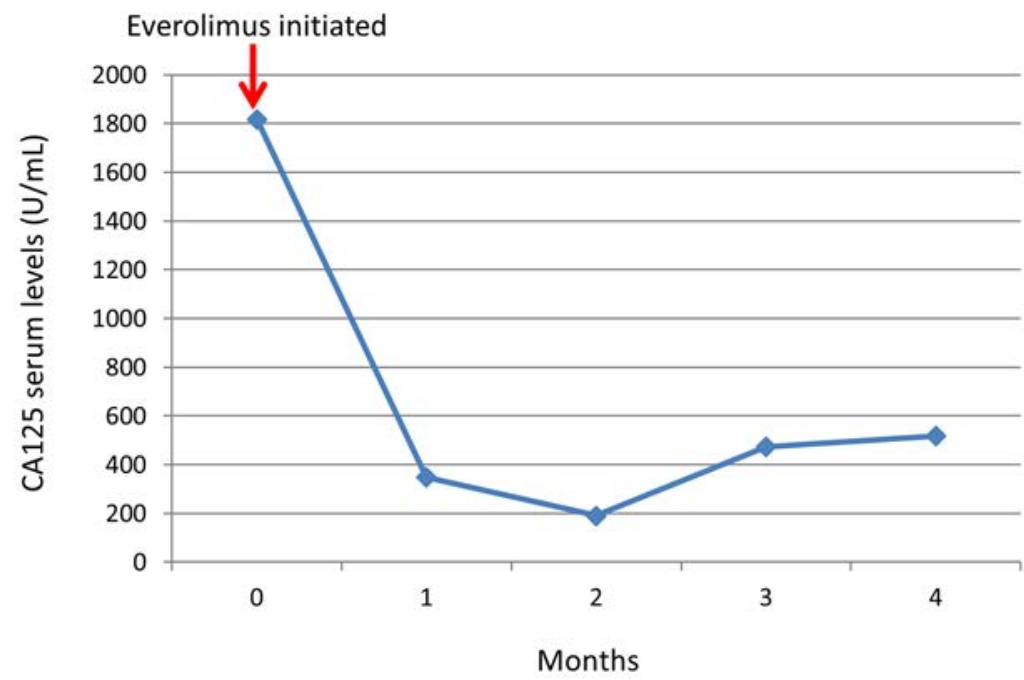

sarcoma following identification of an EWSR1 rearrangement by OncoPanel (30). The identification of an $A L K$ rearrangement in a uterine leiomyosarcoma led to a revised diagnosis of inflammatory myofibroblastic tumor at the time of peritoneal dissemination in case 3 . The patient has not required further treatment to date; however, if therapy is required in the future she will receive an ALK inhibitor rather than standard chemotherapy for leiomyosarcoma. In case 4 , identification of a KIT exon 11 in-frame deletion in a small bowel undifferentiated sarcoma triggered additional immunohis-

tochemistry studies and led to a revised diagnosis of gastrointestinal stromal sarcoma. As a result, the patient was initiated on imatinib therapy, with no evidence of recurrent disease at last follow-up. In case 5, a patient with a history of high-risk gastrointestinal stromal tumor (GIST), identification of a CTNNB1 mutation in a "metastatic peripancreatic GIST," led to reevaluation of the pathology and immunohistochemistry, with revision of the diagnosis to desmoid tumor and maintenance of current therapy.

Most adult carcinomas lack site-specific translocation or mutational events; however, the etiologic associations of hypermutation signatures can provide diagnostic insights. In case 6 , identification of a mismatch repair deficiency signature in a high-grade uterine malignancy suggested a diagnosis of undifferentiated endometrial carcinoma and raised the possibility of Lynch syndrome. In case 7, a patient diagnosed with primary lung squamous cell carcinoma, the presence of a UVA signature indicated that the tumor was actually a metastasis from a cutaneous site.

Patient selection for clinical trial enrollment. Several studies have shown that the frequency of actionable cancer gene alterations follows a "long tail" distribution (13), that is, alterations that may specify response to targeted therapy, and with significant clinical implications, may be seen rarely at individual cancer centers. To explore this hypothesis, we sought to mine our genomic and clinical knowledge base to identify exemplary cases that might inform studies of therapeutic utility in particular genetic contexts.

Tumor suppressor genes as therapeutic targets. A 59-year-old woman with advanced ovarian papillary serous carcinoma was followed over 5 years, during which time she underwent radical cytoreductive surgery and multiple courses of chemotherapy, a clinical trial of olaparib and cediranib, surgical debulking, and ongoing catheter drainage due to ascites. Testing of archival tumor showed biallelic TSC2 inactivation (Figure 3A). The woman was enrolled on a clinical trial of everolimus and had a dramatic response within a few weeks, including a decrease in her ascites from 21 to $60 \mathrm{ml}, \mathrm{CA}-125$ 
Table 4. Tumors with TSC1 or TSC2 mutation and copy number loss

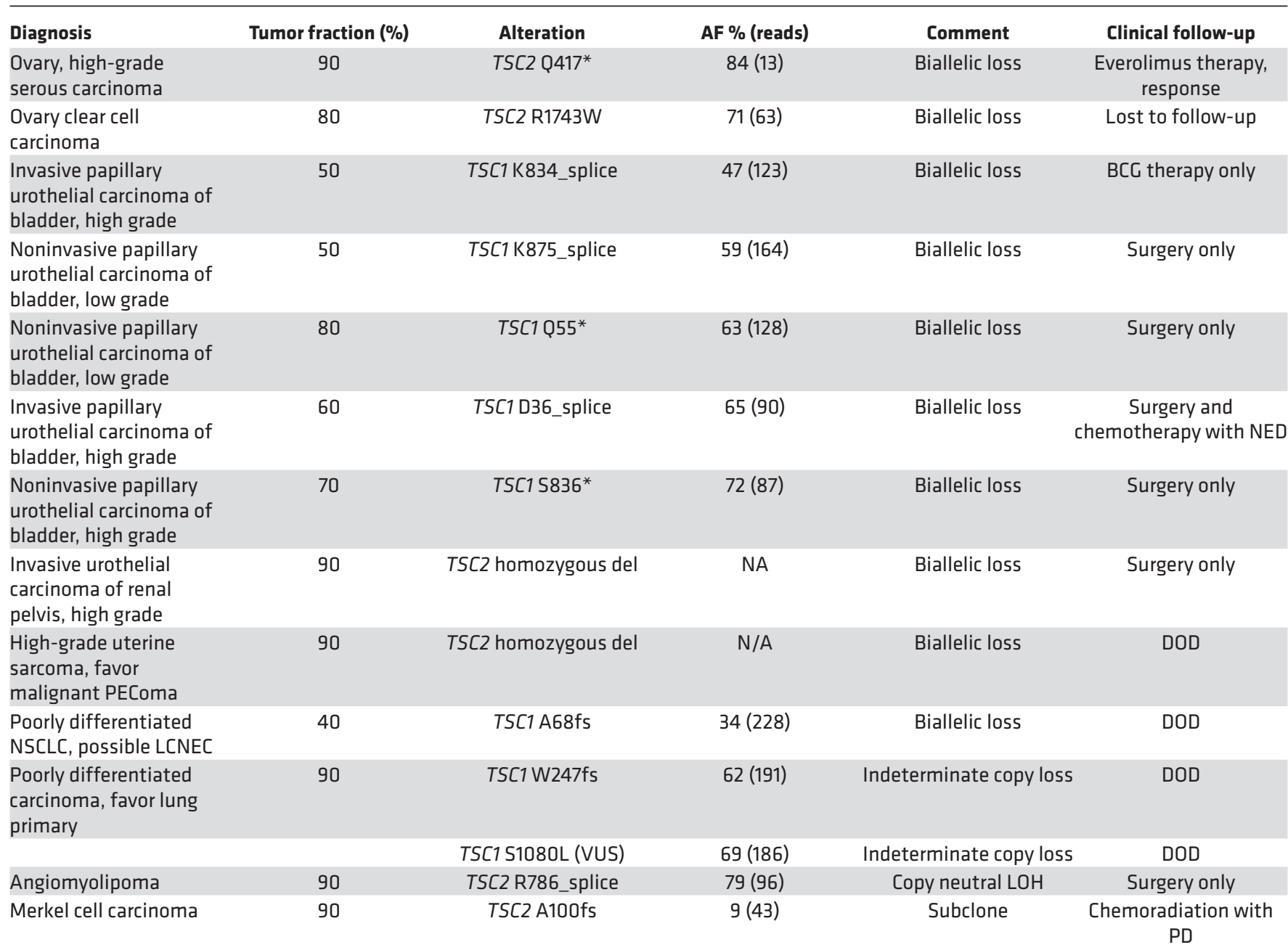

AF, allele fraction; DOD, dead of disease; LCNEC, large-cell neuroendocrine carcinoma; LOH, loss of heterozygosity; NED, no evidence of disease; NSCLC, non-small-cell lung carcinoma; PD, progressive disease; PEComa, perivascular epithelioid cell tumor; VUS, variant of unknown significance.

levels that dropped from 1,816 units/ $\mathrm{ml}$ to 348 units/ $\mathrm{ml}$ after 4 weeks of treatment (Figure 3, B and C), and a decrease in index lesions by $10 \%$ after 2 months. She had substantial improvement in her quality of life and was able to return to work and her normal activities.

The OncoPanel database was analyzed for other tumors with similar evidence of 2-copy TSC2 loss. A total of 56 loss-of-function (LOF) variants in TSC1 or TSC2 were detected across 43 tumors (1.1\%; Supplemental Figure 2). Of these, 22 variants in 10 tumors occurred in hypermutated samples. To reduce the risk of false discovery (31), these were removed from further analysis. Of the 33 remaining, 12 showed evidence for both a LOF coding mutation and loss of one copy of the gene or homozygous gene deletion (Table 4). Using variant allele fraction to exclude predicted subclonal events, 10 ( $0.2 \%$ overall) showed biallelic TSC1 or TSC2 loss, including 6 urothelial carcinomas (representing $5 \%$ of 122 total).

Rare activating ERBB2 mutations in ovarian cancer. A 48-year-old woman with low-grade ovarian serous carcinoma (originally diagnosed age 18) was managed at progression with chemotherapy, followed by multiple phase I clinical trials, including monoclonal antibody therapy against HER3 and combined $\mathrm{MEK} 1 / 2$ and $\mathrm{PI} 3 \mathrm{~K}$ inhibitors, all with symptomatic and radiographic progression. Analysis of her recurrent tumor identified an ERBB2 Tyr772_Ala775dup mutation (Figure 4A), which prompted off-label use of trastuzumab and navelbine with symptomatic improvement and radiographically stable disease for 21 months at last follow-up. 
A

Low grade serous carcinoma with ERBB2 Tyr772_Ala775dup

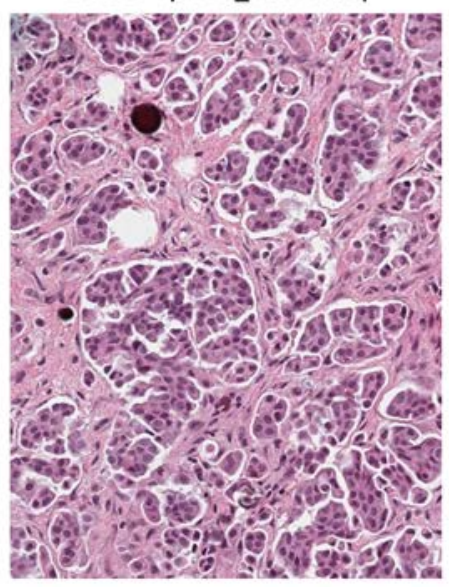

B

Mutational profile of low grade serous carcinomas

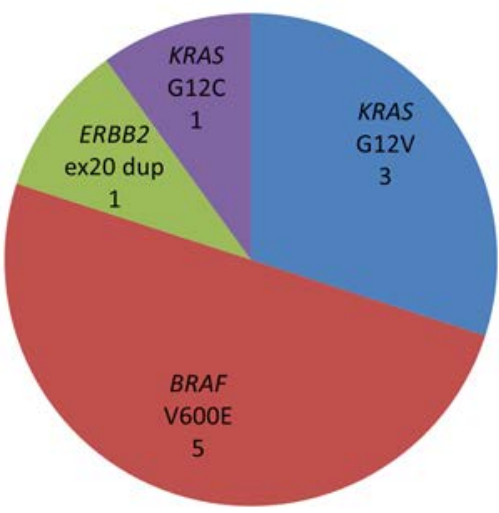

Figure 4. Achieving disease control using ERBB2-targeted therapy in low-grade serous carcinoma. (A) Peritoneal biopsies in a 48-year-old patient with a longstanding history of low-grade serous carcinoma show a monomorphic proliferation of epithelial cells with bland cytologic features and frequent psammoma bodies (original magnification, $\times 400$ ). Tumor genotyping revealed an ERBB2 duplication mutation in exon 20. (B) Ten cases of low-grade serous tumors were sequenced: a mean of 3 single nucleotide variants (SNV) (2.4 SNVs per Mb) per case was seen. Two KRAS G12V-mutated cases showed an additional activating PIK3CA mutation; one KRAS G12V-mutated case showed an additional BRAF D594C mutation. Notably, none had concurrent mutations in tumor suppressor genes.

Low-grade ovarian serous carcinomas in this cohort were characterized by mutations in $K R A S, B R A F$ V600E, or $E R B B 2$ and a few other genomic alterations (Figure 4B), raising the possibility that the prolonged disease control with ERBB2 inhibitors observed in this patient's tumor may be attributable to single-pathway dependency. Known activating ERBB2 mutations were detected in 49 cases (1.3\% of the OncoPanel cohort) and were enriched in lung, bladder, and low-grade gynecologic tract tumors (Table 5 and 6 ). All 6 endometrial adenocarcinomas with activating $E R B B 2$ mutations occurred in the context of hypermutation. In breast, colon, and gastric cancers, ERBB2 mutations co-occurred with activating mutations in $K R A S, B R A F$, and $E G F R$; whether this reflects tumor heterogeneity or simultaneous driver events in the same clone is unknown, though the mutant allele fractions did not suggest subclonal events. ERBB2 activating mutations were the only clear functional alterations in a subset of tumors, most commonly lung adenocarcinomas, as well as in a case of spinal cord neurofibroma and soft tissue schwannoma. This genomic context may lend some insight into the relative likelihood of success of targeted inhibition of ERBB2 mutations in different tumor types (32).

An EGFR splice mutation associated with erlotinib response. A 59-year-old male never smoker presented with lung adenocarcinoma metastatic to bone and brain. The patient underwent resection of the brain mass and radiation to the brain and bony metastases. Genotyping was negative for common EGFR mutations and $A L K$ FISH was negative. He was started on first-line cisplatin/pemetrexed therapy. After 5 months, brain MRI showed asymptomatic slow progression. The brain metastasis was profiled, revealing an unusual EGFR intron 19 splice mutation (EGFR c.2283+1G>A [p.D761_splice] and c.2283+12_149del) in addition to high-level amplification of EGFR (Figure 5A). Based on its location within the splice site sequence, this variant is predicted to abrogate the canonical splice site, leading to read through to an alternative splice donor site in the intron that would result in a 4-amino acid insertion that translates to A763_Y764insMSSW. Other insertions reported at this site are associated with sensitivity to EGFR inhibitors, unlike most other EGFR exon 20 insertion mutations (33). Although this particular EGFR alteration has not been classified as tyrosine kinase inhibitor (TKI) sensitizing, the patient was started on $150 \mathrm{mg}$ erlotinib daily, with reduction in the lung mass on initial restaging (Figure 5A). The brain metastases progressed after 5 months, after which the patient was treated with whole-brain radiation therapy. He experienced durable extracranial response on erlotinib for a total of 12 months before developing systemic progression in bone and lung (34). When erlotinib was stopped in preparation for subsequent therapy, systemic disease rapidly progressed. The patient was refractory to further therapy and died 2 months after stopping erlotinib.

Amplification of AXL associated with response to an Axl kinase inhibitor. A 56-year-old male, never smoker, was diagnosed with metastatic lung adenocarcinoma, with bilateral mediastinal lymphadenopathy and pleural carcinomatosis. Genomic analysis demonstrated focal gain of 19q12-13.2, including CCNE1, AKT2, and $A X L$ as well as $C D K N 2 A$ / $B$ loss. The tumor was wild-type for $E G F R, A L K, R O S 1, R A S$, and MET. He was treated with carboplatin/pemetrexed and bevacizumab, followed by docetaxel, with a best response of stable disease. Based on CCNE1 gain, he was enrolled in a clinical trial combining an ATR inhibitor with cisplatin. His course was complicated by pneumonia and continued disease progression. The mean $\log 2$ copy ratio for $A X L$ was 0.94 , and tumor purity based on pathologist's review was estimated at $60 \%$, with approximately 5 normalized copies 
Table 5. Tumors with ERBB2 activating mutations across the cohort with clinical follow-up

\begin{tabular}{|c|c|c|c|c|c|c|c|c|}
\hline Diagnosis & $n(\%)^{A}$ & Other tier 1-3 alteration & HER2 TX & Standard TX & $\begin{array}{c}\text { Other clinical } \\
\text { trial }\end{array}$ & Surgery only & DOD & Lost to $f / u$ \\
\hline Lung & $13(3)$ & & & & & & & \\
\hline ADC stage I & & $E R B B 2 \mathrm{amp}$ & & & & 1 & & \\
\hline ADC stage I & & None & & & & 1 & & \\
\hline ADC stage IIIA & & None & & 1 & & & & \\
\hline ADC stage IV & & ERBB2 amp & & & & & & \\
\hline ADC stage IV & & None & 1 & & & & & \\
\hline ADC stage IV & & None & 1 & & & & & \\
\hline ADC stage IV & & None & & 1 & & & & \\
\hline ADC stage IV & & None & & & & & 1 & \\
\hline ADC stage IV & & None & & & & & 1 & \\
\hline HG PUC stage I & & ERBB2 as subclone & & & & & & 1 \\
\hline HG PUC stage I & & TP53, hypermutated & & & & & & 1 \\
\hline HG PUC stage III & & TP53 & & & & 1 & & \\
\hline HG PUC stage IV & & RB1, PIK3CA & & & & & 1 & \\
\hline HG PUC stage IV & & $R B 1$ & & 1 & & & & \\
\hline HG PUC stage IV & & $R B 1$ & & & 1 & & & \\
\hline HG PUC stage IV & & TP53 & & & & & 1 & \\
\hline HG PUC stage IV & & $\begin{array}{l}\text { PIK3CA, PTEN, } \\
\text { hypermutated }\end{array}$ & & 1 & & & & \\
\hline HG PUC stage IV & & $\begin{array}{c}\text { CDKN2A and CDKN2B } \\
\text { del }\end{array}$ & & 1 & & & & \\
\hline EMC grade 3 & & POLd signature & & & & 1 & & \\
\hline EMC grade 3 & & MMRd signature & & 1 & & & & \\
\hline Mixed EMC/serous & & TP53, KRAS, NF1 & & 1 & & & & \\
\hline Cervix, SCC & $1(3)$ & PIKЗСA & & 1 & & & & \\
\hline $\begin{array}{l}\text { Ovary, low-grade } \\
\text { serous CA, recurrent }\end{array}$ & $1(10)$ & None & & 1 & & & & \\
\hline Total & $32(1.3)$ & & 3 & 9 & 1 & 9 & 6 & 3 \\
\hline
\end{tabular}

APercentage of tumors with this diagnosis. ${ }^{B}$ Tumor content was at the minimum accepted, therefore other alterations cannot be excluded. ADC, adenocarcinoma; amp, amplification; CA, carcinoma; del, deletion; DOD, dead of disease; EMC, endometrial adenocarcinoma; f/u, follow-up; HG PUC, high-grade papillary urothelial carcinoma; MMRd, mismatch repair deficient; POLd, polymerase defect; TX, treatment.

relative to an assumption of diploidy (see Methods). He was enrolled on a phase I clinical trial of MGCD265, a TKI targeting Met and Axl. Within a few days of starting treatment, he had a dramatic response and no longer required oxygen support; he was able to bike 7 miles a day after 1 month of treatment. Restaging scans after 8 months of treatment demonstrated a $66 \%$ decrease in index lesions (Figure 5B).

Based on this response, we investigated further $A X L$ amplification events. A similar level of copy gain (approximately 3-6 copies), minimally including $A X L$ but often extending to $C C N E 1$, was identified in 7 cases ( $0.2 \%$ of our population; Table 7 ). High copy gain of $A X L$ (copy number $>6$ ) was detected in one case in the cohort $(0.02 \%)$ - an endometrial serous carcinoma with approximately 12 copies. 
A

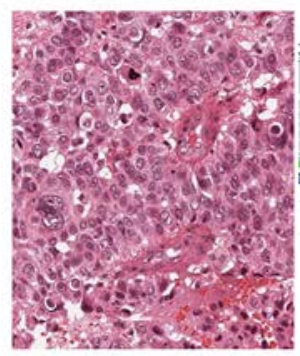

At start of erlotinib therapy

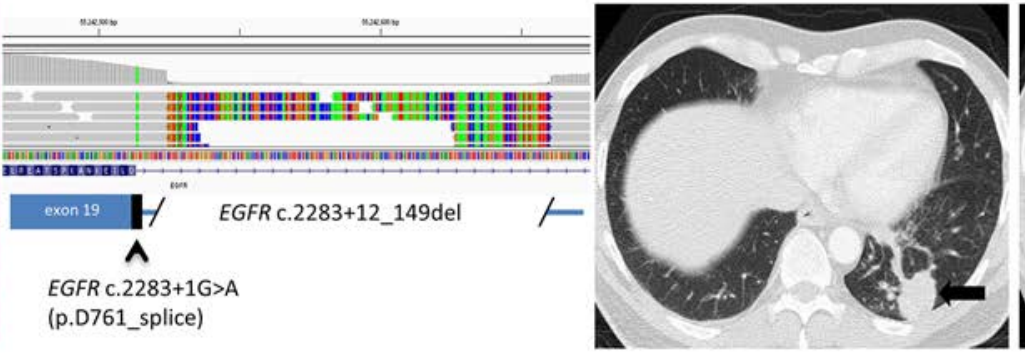

19q12-13.11 focal gain:

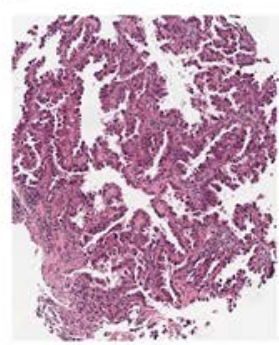
CCNE1, RHPN2, CEBPA, AKT2, AXL

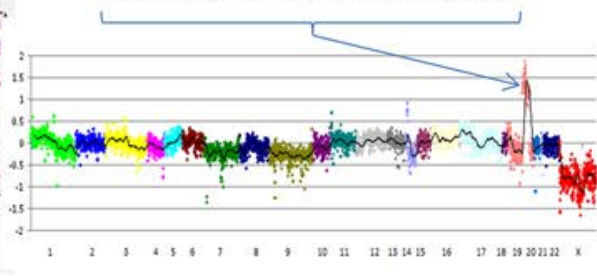

Pre-treatment

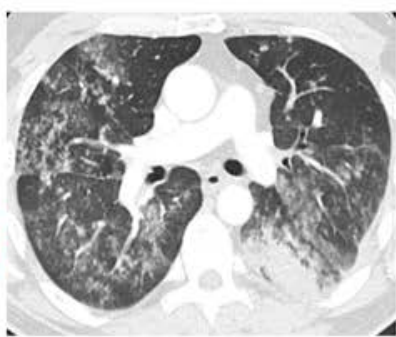

12 week followup

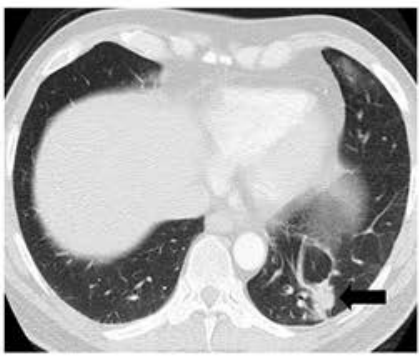

2 Months on Treatment

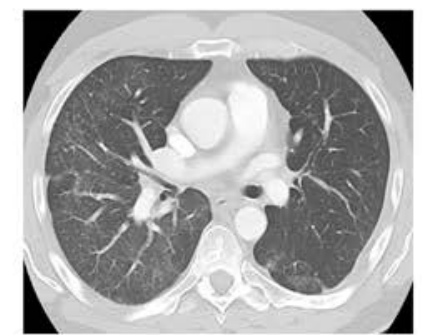

Figure 5. Clinical effect of genomic profiling. (A) A nonsmoking man with metastatic lung adenocarcinoma to the brain (original magnification, $\times 400$ ) had a complex EGFR intron 19 mutation involving the splice junction (EGFR c.2283+1G>A [p.D761_splice] and c.2283+12_149del) in 92\% of 780 sequencing reads. The read count is consistent with high-level amplification of the mutated EGFR allele. Baseline chest CT prior to the initiation of erlotinib therapy demonstrated a 3.8-cm mass in the left lower lobe (arrow). On a follow-up chest CT at 12 weeks of erlotinib therapy, the mass has decreased in size, measuring $2.5 \mathrm{~cm}$ (arrow), representing response to therapy. (B) A nonsmoking man with lung adenocarcinoma (original magnification, $\times 100$ ) with pleural carcinomatosis had focal gain of chromosome 19q12-13.11, including AXL. Baseline chest CT prior to initiation of MET-AXL inhibitor therapy demonstrated bilateral lung nodules and ground glass infiltrates. Follow-up chest CT at 2 months shows near-complete resolution of the lung infiltrates.

We used publicly available data from The Cancer Genome Atlas (TCGA) $(35,36)$ to further evaluate the frequency of $A X L$ amplification across 11,613 tumors representing 34 distinct diseases. We included any tumor with an $A X L \log 2$ ratio of $\geq 0.36$ (i.e., corresponding to a single-copy gain in a tumor of at least $60 \%$ tumor content), filtering to exclude whole chromosome or arm-level copy number changes. Focal amplifications of $A X L$ were identified in $0.65 \%(n=76)$ of tumors (Supplemental Figure 3$)$; high-level amplifications were uncommon $(n=3)$, with an average size of $1.52 \pm 1.23 \mathrm{Mb}$, and identified exclusively in ovarian serous carcinomas. Low-level amplifications occurred in $0.63 \%(n=73)$, including in ovarian cancer $(n=19)$, sarcoma $(n=13)$ lung squamous cell carcinoma $(n=11)$, breast cancer $(n=$ 8 ), and lung adenocarcinoma $(n=3)$. Axl activation has also been reported as a mechanism of resistance following EGFR TKI therapy in lung cancer, with preclinical studies suggesting a therapeutic role for Axl inhibitors in this setting; in our cohort, we identified one such case (Table 7) (37-39).

In the first year of study, 50 patients $(n=50)$ had OncoPanel testing initiated by clinicians in the Early Drug Development Center, the facility for phase I clinical trial enrollment at DFCI. Because patient selection for many of these phase I trials requires a genomic biomarker, we looked at the frequency of potentially actionable variants in this cohort and clinical trial enrollment. The patients in this cohort had a variety of cancer diagnoses (Supplemental Table 7). 43 OncoPanel reports were generated (7 had insufficient material for testing). 31 of $50(62 \%)$ patients had at least one tier 2 single nucleotide variation or copy number variant detected, similar to the results of our cohort overall. In this setting, $16(32 \%)$ of these patients - just over half (52\%) of those with an actionable or informative result and $32 \%$ of the full group — were enrolled on a genomic-based clinical trial (Supplemental Table 7).

\section{Discussion}

In recent years, a number of academic cancer centers, hospitals, and commercial entities have established genomic profiling programs $(11,12,16,17,20,24,26,40)$ that differ in terms of technology and gene panel content (varying from tens to hundreds or thousands of genes) as well as target patient populations (specifying cancer type or stage of disease, for example) $(16,21)$ but are similar in that they apply multigene testing 
Table 6. Tumors with ERBB2 activating mutations across the cohort with clinical follow-up

\begin{tabular}{|c|c|c|c|c|c|c|c|c|}
\hline Diagnosis & $n(\%)^{A}$ & $\begin{array}{l}\text { Other tier 1-3 } \\
\text { alteration }\end{array}$ & HER2 TX & Standard TX & $\begin{array}{c}\text { Other clinical } \\
\text { trial }\end{array}$ & Surgery only & DOD & Lost to $f / u$ \\
\hline \multicolumn{9}{|l|}{$\begin{array}{l}\text { Gastrointestinal } \\
\text { tract }\end{array}$} \\
\hline Colon & $4(2)$ & & & & & & & \\
\hline ADC & & $B R A F(V 600 E)$ & & & & & & 1 \\
\hline$A D C$ & & KRAS, TP53 & & & & & 1 & \\
\hline$A D C$ & & TP53 & & 1 & & & & \\
\hline ADC & & TP53 & & 1 & & & & \\
\hline Anus SCC & $1(10)$ & РІКЗСА & & & 1 & & & \\
\hline \multicolumn{9}{|l|}{ Stomach } \\
\hline Signet ring ADC & $1(3)$ & $\begin{array}{l}\text { KRAS, EGFR } \\
\text { (L861Q), CDH1 }\end{array}$ & & & & & 1 & \\
\hline $\begin{array}{l}\text { Upper GI } \\
\text { unknown } \\
\text { primary ADC }\end{array}$ & $1(14)$ & $\begin{array}{l}\text { CTNNB1, PTEN, } \\
\text { hypermutated }\end{array}$ & & & & & 1 & \\
\hline Pancreas ADC & $1(4)$ & ERBB2 amp & & & & & 1 & \\
\hline Breast & $4(2)$ & & & & & & & \\
\hline $\begin{array}{l}\text { PD IDC, ER+, } \\
\text { stage IV }\end{array}$ & & РІКЗСА & & & & & 1 & \\
\hline $\begin{array}{l}\text { PD IDC, ER+, } \\
\text { stage IV }\end{array}$ & & TP53 \& BRCA1 & & & 1 & & & \\
\hline TNBC, stage IV & & $\begin{array}{c}\text { KRAS, } \\
\text { PIKЗСA,TP53 }\end{array}$ & & & & 1 & & \\
\hline $\begin{array}{l}\text { ILC, } \mathrm{ER}^{+} \text {, stage } \\
\text { IV }\end{array}$ & & $\mathrm{CDH1}$ & & & & 1 & & \\
\hline \multicolumn{9}{|l|}{ Other } \\
\hline $\begin{array}{l}\text { Spinal cord } \\
\text { neurofibroma }\end{array}$ & 1 (100) & None & & 1 & & & & \\
\hline $\begin{array}{l}\text { Soft tissue } \\
\text { schwannoma }\end{array}$ & $1(100)$ & None & & & & 1 & & \\
\hline $\begin{array}{l}\text { Skin apocrine } \\
\text { CA }\end{array}$ & $1(3)$ & TP53 & 1 & & & & & \\
\hline $\begin{array}{l}\text { Head and neck } \\
\text { HPV }^{+} \text {SCC }\end{array}$ & $1(0.6)$ & ERBB2 amp & 1 & & & & & \\
\hline Total & $16(1.3)$ & & 2 & 3 & 2 & 3 & 5 & 1 \\
\hline
\end{tabular}

to cancer patients, with a goal of more effectively triaging those patients to the appropriate therapeutic regimen - whether on-label, off-label, or experimental/clinical trial. In most cases, a categorization strategy is applied to the genomic profile in order to obtain a shortlist of potentially "actionable" or "informative" alterations that can be used to tailor treatment options in an individualized manner. Depending on the number of genes tested, and the strategy for categorizing such events, the proportion of patients that have a potentially actionable alteration varies but is in the $75 \%-90 \%$ range. While encouraging, the proportion of patients that actually have this information used for clinical management remains much lower, in the $5 \%-10 \%$ range. There are a multitude of reasons for this, ranging from patient preferences (20) and physician comfort with genomic data (22), to biologically relevant information, such as time of testing in the course of a patient's disease, availability of clinical trials, and cost of off-label use of drugs. Despite these barriers, there is evidence to support the clinical utility of molecularly targeted therapy in specific indications $(4,21,24,25)$, though heavily pretreated patients may not benefit to the same degree (25).

We report a large multi-institution clinical sequencing effort using a several-hundred-gene assay that profiles a broad, unselected cancer patient population, spanning adult and pediatric patients, early- and late-stage 
Table 7. Pathology and follow-up for tumors with AXL copy number gain

\begin{tabular}{|c|c|c|c|c|c|c|}
\hline Diagnosis & Tumor fraction (\%) & $A X L \mathbf{C N}$ & Genes in amplicon & Other alterations & CNI & Clinical follow-up \\
\hline $\begin{array}{l}\text { Primary peritoneal } \\
\text { high-grade serous } \\
\text { carcinoma }\end{array}$ & 80 & 3.3 & $\begin{array}{c}\text { CCNE1, RHPN2, } \\
\text { CEBPA, AKT2, AXL }\end{array}$ & $\begin{array}{c}\text { TP53 P278L, PTEN } \\
\text { Q171*A , PTEN G209*A }\end{array}$ & $\mathrm{Y}$ & $\begin{array}{c}\text { Failed PTEN } \\
\text { inhibitors, } \\
\text { progressive disease } \\
\text { on chemotherapy }\end{array}$ \\
\hline $\begin{array}{l}\text { Follicular thyroid } \\
\text { carcinoma, } \\
\text { angioinvasive }\end{array}$ & 90 & 3.4 & $\begin{array}{c}\text { CCNE1, RHPN2, } \\
\text { CEBPA, AKT2, AXL }\end{array}$ & TP53 R213Q & $\mathrm{N}$ & $\begin{array}{l}\text { Li Fraumeni } \\
\text { syndrome, NED at } 1 \\
\text { year follow-up }\end{array}$ \\
\hline $\begin{array}{l}\text { Lung } \\
\text { adenocarcinoma }\end{array}$ & 80 & 4.2 & $\begin{array}{c}\text { RHPN2, CEBPA, } \\
\text { AKT2, AXL }\end{array}$ & $\begin{array}{l}\text { EGFR L858R, EGFR } \\
\text { T790M, CCND1 amp }\end{array}$ & $\mathrm{N}$ & $\begin{array}{l}\text { Progressed through } \\
\text { AZD9291 and } \\
\text { pemetrexed therapy }\end{array}$ \\
\hline $\begin{array}{l}\text { Female genital tract } \\
\text { carcinosarcoma }\end{array}$ & 80 & 5.2 & $A K T 2, A X L$ & TP53 P278S & $\mathrm{Y}$ & $\mathrm{DOD}$ \\
\hline $\begin{array}{l}\text { Endometrial serous } \\
\text { carcinoma }\end{array}$ & 60 & 12 & CCNE1, AKT2, AXL & TP53 V274F & $\mathrm{Y}$ & $\begin{array}{c}\text { Progression on } \\
\text { chemotherapy, DOD }\end{array}$ \\
\hline
\end{tabular}

${ }^{A}$ PTEN variants present at subclonal levels (4\%-8\% allelic fraction). amp, amplification; CN, copy number; CNI, copy number instability; del, deletion; DOD, dead of disease; NED, no evidence of disease.

disease, solid tumors, and hematologic malignancies. Our cohort is reflective of the full spectrum of patients seen at our institutions but also incorporates biases in terms of the ordering physician, perceived utility of the test in a disease-specific context, and the availability of specimen to be tested. All genomic data generated in our CLIA-certified laboratory is deposited into a research knowledge base that enables linkage to clinical information and will enable future studies as well as enabling the prospective identification of patients for genomically matched clinical trials (Figure 6).

Our data show that, across an unselected cancer cohort, genomic profiling in a CLIA-certified laboratory is technically feasible. This requires rigorous validation of the lab chemistries and the development and implementation of comprehensive analytical algorithms, followed by manual review and signout by a pathologist. Our OncoPanel test performs with high sensitivity, specificity, accuracy, and reproducibility for the detection of mutations, copy number changes, and structural rearrangements.

Because we introduced an updated gene menu and sequencing chemistries after 1 year of sequencing, we have chosen to restrict our genomic and correlative analyses to data collected in that first year; however, many features of the operation have remained steady since 2013-2014. The consent rate for patients across our institutions is approximately $70 \%$. Over the course of sequencing more than 12,000 samples to date, we have observed a $74 \%$ rate of available pathology material, a $59 \%$ rate of adequacy for sequencing, and a $56 \%$ rate overall of generating a sequencing report with a $95 \%$ sequencing success rate. These metrics are similar, albeit with a somewhat higher rate of specimen retrieval and histologic adequacy overall as compared with the first year of testing (Figure 1B). Overall, just over half of patients with consent and a test order receive a result. The largest barrier to obtaining adequate tumor tissue for sequencing under this research protocol was lack of access to many patient samples that were located at outside institutions and were not actively retrieved for OncoPanel testing. We anticipate that moving toward clinical testing should significantly improve our ability to obtain and test tumor specimens; once sufficient material was received into the laboratory for pathology review, $73 \%$ were scored as sufficient for genomic profiling. Advances in alternative testing approaches, including testing of circulating tumor cell-free DNA, should bring genomic testing to a broader population of patients lacking adequate tissue biopsy samples. The median turnaround time for a result was 5.3 weeks after receipt of sample - this is reasonable for a research test but not a clinical assay. Streamlining processes and making improvements in lab chemistries and analytical pipelines will 


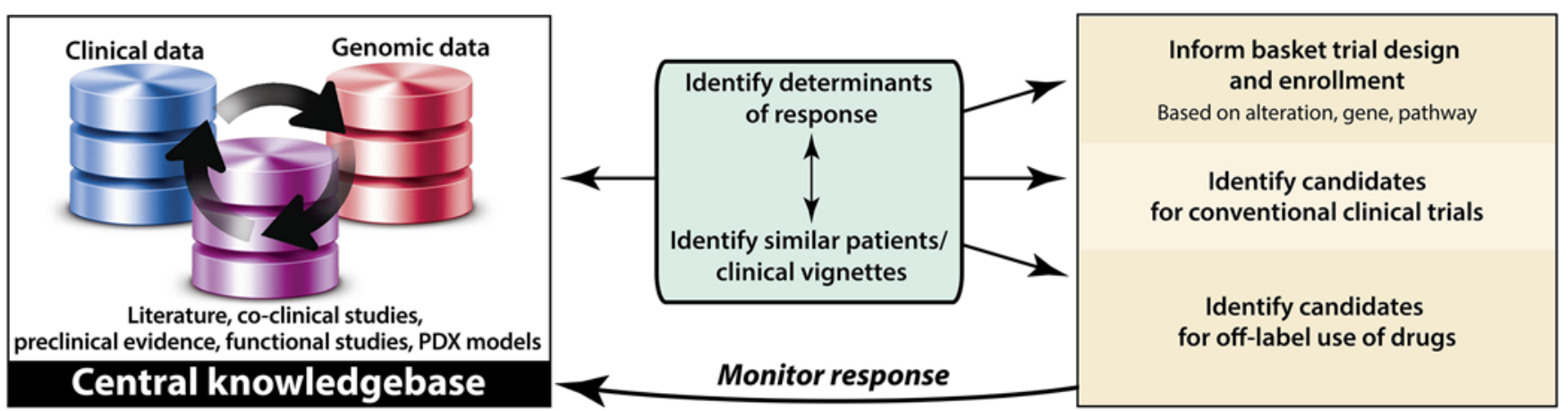

Figure 6. A knowledge base is key to maximizing the effect of genomic testing on precision medicine. Genomic data are tiered according to clinical actionability based on available evidence, incorporating published literature and supporting evidence from preclinical and functional studies, and feed into a central knowledge base that interfaces with clinical data repositories. These data can be queried as part of IRB-approved clinical trials and research studies and used to identify determinants of response as well as patients with similar genomic profiles that may be candidates for targeted therapies. Going forward, this mechanism can also be used to enable more rational design of basket trials.

allow us to achieve a more reasonable clinical timeframe for the next phase of our program.

Biologically, broad genomic profiling across cancers builds upon the results of large, multi-institutional research studies, like TCGA and International Cancer Genome Consortium, and also enables the identification of potentially actionable alterations in rarer cancers. Unique to this cohort is the fact that few restrictions were imposed on the time (in course of disease, grade, or stage) at which genomic testing was ordered, which allows us to broadly compare genomic profiles of primary and metastatic disease. Our data suggest that for certain tumor types, patterns of actionable genomic alterations may vary between primary and metastatic sites and raise the possibility that different information may be gleaned from different sites of disease. Future studies examining serial biopsies from individual patients should further clarify the clinical significance of genetic heterogeneity.

In this study, we demonstrated that enterprise-level genomic profiling was technically feasible and useful for retrospective research purposes. The ultimate goal of implementing such a program, however, is to determine clinical utility - i.e., to improve outcomes for cancer patients. As this was a research test (rather than a clinical trial), we are not systematically able to evaluate the clinical impact of genomic profiling; rather, our analyses are anecdotal. Nonetheless, these "exceptional responder" cases allow us to make new observations about driver alterations in cancer, and retrospective analyses of similar genomic events allow us to make inferences about larger patient cohorts and additional challenges that need to be addressed to maximize utilization of precision cancer medicine.

Although our sequencing program was not originally conceived as a diagnostic service, the unbiased approach to tumor genomic analysis uncovered multiple instances in which sequence-level findings revealed genomic changes that are characteristic of certain tumors not originally considered in the pathologic differential diagnosis and led to clarified or revised diagnoses. The frequency with which sequencing can be expected to lead to a change in diagnosis is difficult to estimate from this study, as we did not systematically capture these types of events. However, it is clear that this type of assay can bring added diagnostic value, even in a setting in which expert pathology review is standard. Furthermore, the results of implementing profiling across all cancer types provide evidence that the systematic use of broad-scale genomic sequencing enables precision therapeutics and is likely to be highly valuable at institutions that offer a broad portfolio of clinical trials. An exemplary finding in our cohort was the presence of biallelic TSC1/TSC2 inactivation in a small but appreciable percentage of bladder carcinomas, consistent with previous reports of response to everolimus $(41,42)$, indicating that these should be taken into account in the design of mTOR inhibitor trials, including ongoing basket trials (as is the case in our institution).

More strikingly, our comprehensive testing strategy led to the detection of uncommon and unanticipated driver events. For example, we found an EGFR intron 19 splice mutation in lung adenocarcinoma associated with a modest but prolonged response to erlotinib therapy; further investigation is needed to determine the degree to which EGFR splice variants contribute to EGFR activation and TKI sensitivity in patients with lung adenocarcinomas. In another example, we identified a case of 
amplification of the $A X L$ tyrosine kinase gene in the absence of other known driver alterations in lung adenocarcinoma associated with clinical response to an Axl inhibitor. The rarity of this alteration in both our cohort and TCGA raises the issue of identifying sufficient numbers of a genotype to enable clinical trials. One possible solution is enabling multi-institutional basket trials and sharing of genomic data to identify such patients - an endeavor that has been endorsed by the American Association for Cancer Research in Project GENIE, to which we are a contributing institute. Incorporation of complementary technologies, such as RNA sequencing, may be particularly fruitful when seeking targets such as Axl that are subject to epigenetic or RNA-based regulation (39).Finally, our analysis provides some understanding of the feasibility and actionability of systematic cancer profiling of a large, unselected patient population. Analysis of the frequency of gene alterations across all tumor types suggests that over two-thirds of tested tumors have some potentially targetable alteration. This agrees with previously published studies that found a similarly high proportion of patients with potentially actionable alterations $(64 \%-90 \%$, refs. $4,18,19)$, though it has also been reported that a much smaller proportion of patients $(5 \%-11 \%)$ are actually treated based on the mutational profile or enrolled on a clinical trial $(16,19,20)$. Our focused examination of patients with targetable TSC1/2 or ERBB2 or AXL alterations suggests that there are patients with actionable alterations in these select targets across disease types, but only approximately $10 \%$ had their genomic profile used for clinical management (1 of 13 TSC1/2 alterations [Table 4]; 5 of 49 ERBB2 activating mutations [Tables 5 and 6]; 1 of 8 AXL copy gain [Table 7]; and total 7 of 70 cases in which genomic profile was used clinically). We identified several reasons for the low rates of trial enrollment, including standard of care therapy providing effective disease control, death due to disease before genomically driven therapy could be considered, or enrollment in a clinical trial not related to the genomic results. Specific to the research nature of this program, our cohort contains many early-stage patients that require no further therapy beyond surgical resection (Tables 4 and 5). In addition, certain limitations on return of results imposed by the research protocol meant that some potentially actionable alterations in patients' cancers could not have clinical impact, suggesting the importance of migrating our research genomic test to a routine clinical test. In a chart review of 50 advanced cancer patients who had an OncoPanel test ordered in the phase I clinical trials group, $16(32 \%)$ were found to have changes in recommendation for trial options, though additional factors (such as progressive disease) must be considered before a patient is enrolled on a trial. Although the number of patients analyzed was small and the data are context specific, this demonstrates that the rate of genomically driven enrollment in this setting is relatively high, reflecting the specific expertise of this group of oncologists and the access to a portfolio of genomic-based trials, and indicates that the combination of comprehensive molecular testing and ready access to genomically driven trials can improve rates of clinical trial enrollment.

There are many open questions regarding the appropriate scope and scale of cancer genome profiling in the clinical setting, particularly in relation to the decreasing cost of DNA sequencing. One consideration is the comparison of whole-exome sequencing or whole-genome sequencing to more targeted gene panels. A major advantage of gene panels - as opposed to whole-exome sequencing or whole-genome sequencing — is the option to sequence without a matched normal. While tumor-only sequencing is more feasible for logistical (i.e., ability to obtain a matched normal specimen for each patient) and financial (cost of performing more sequencing) reasons, we recognize that there are limitations in the detection of relevant cancer-susceptibility germline events, among others (43). To address this issue, we developed an informatics pipeline to filter common single nucleotide polymorphisms present in public and internal noncancer populations; to incorporate data on the allelic fraction (with contextual purity and ploidy information) of a mutation; and to apply a tiering strategy to the interpretation of alterations. In a study of a subset of 91 cases comparing our filtering approach, including pathologist review, with matched tumor-normal sequencing (44), 54 germline variants were reported - i.e., not filtered out by our pipeline. 93\% of these (50 of 54) were reported as tier 4 "variants of unknown significance," 3 were reported as tier 3, and only 1 was reported as a tier 2 result. This resulted in "false somatic" calls reported as tier 3 or higher in 4 of 91 (4.4\%) of patient samples. This finding is in keeping with the rate of significant occult germline variants found in cancer patients undergoing paired tumor-normal sequencing at other institutions (45).

In practice, we report potential germline variants conservatively, according to clinical relevance. Where there was a suspicion of germline status of a pathogenic allele, the data were communicated to 
the ordering physician with a recommendation to correlate with additional medical history and confirmatory testing, if deemed appropriate. We acknowledge that incorporating matched normal sequencing would allow the definitive ascertainment of somatic versus germline status.

Although our initiative was not designed to measure clinical outcomes explicitly, it nonetheless lays the groundwork for more systematic study of the effect of genomics on clinical practice and patient outcomes. Indeed, this initiative has enabled a large number of other more focused studies of common and uncommon tumor types and, in particular, has permitted prospective identification of relatively uncommon genomic variants to facilitate clinical trial enrollment and biomarker studies (46-54). Migrating to a clinical test, identifying when in the course of a patient's disease genomic profiling should be used, and triaging patients in real-time for clinical trial enrollment should contribute to determining clinical utility on a broader scale. Profile enlivens a framework (see Figure 6 for schematic) whereby individual patient experiences can be extrapolated to systematic investigations on genomic correlation with cancer outcomes that lead to new avenues of scientific inquiry, challenge assumptions about cancer diagnosis, and enable extension of novel and more effective treatment approaches to new sets of patients.

\section{Methods}

See the Supplemental Methods, and Supplemental Tables 1, 8 and 9, for details on study design, patient recruitment, assay design and technical validation, data analysis and statistics, and clinical use.

Statistics. Categorical comparisons were performed using Fisher's exact or Chi square tests with Bonferroni correction for multiple comparisons. Sample means were compared using a 2-tailed Student's $t$ test assuming equal variance. $P$ values of $<0.05$ were considered significant. Sensitivities and specificities with $95 \%$ confidence intervals were calculated using a publicly available clinical calculator (vassarstats.net). Confidence intervals were calculated using the Hmisc library in R using the binconf() function set to the "Wilson" calculation method.

Study approval. This study was performed with approval from the DFCI IRB (DFCI IRB protocol 11-104). Written informed consent was obtained from participants prior to inclusion in this study

\section{Author contributions}

LMS, MM, LAG, NIL, and LEM wrote the manuscript. WCH, PWK, BEJ, MM, LAG, BJR, NIL, and LEM designed the study. LMS, PS, EC, AMD, FCK, EPG, YJ, LLR, VRR, PD, AHB, AHL, NIL, JDC, and LEM analyzed the data. RPA, TJP, PVH, FCK, ART, and MD created the informatics pipeline and internally developed software tools. KD, KAJ, AC, MH, SR, JMC, UM, GRO, DJK, MN, and GIS contributed clinical data. RC, VT, and JC supported the MGCD265 clinical trial.

\section{Acknowledgments}

The authors would like to thank Mark Awad, Christopher Fletcher, Suzanne George, and Andrew Wagner for their clinical insights; Jeffrey Golden, Janina Longtine for program support; Sam Hunter, Bernard Fendler, Larry Chung, and Chesley Leslin for bioinformatics contributions; Nikhil Wagle for assistance with panel design; and Emanuele Palescandolo, Dimity Hall, Ling Lin, and Profile technologists for technical expertise. M. Nishino has a research grant from Canon Inc. and grant support from the National Cancer Institute (5K23CA157631). This work was supported by DFCI, BWH, and the National Cancer Institute (5R33CA155554 and 5K23CA157631).

Address correspondence to: Laura E. MacConaill, Dana-Farber Cancer Institute, 450 Brookline Avenue, Dana 1539, Boston, Massachusetts 02215, USA. Phone: 617.582.7754; E-mail: Laura_macconaill@dfci.harvard.edu.

1. Cobleigh MA, et al. Multinational study of the efficacy and safety of humanized anti-HER2 monoclonal antibody in women who have HER2-overexpressing metastatic breast cancer that has progressed after chemotherapy for metastatic disease. J Clin Oncol. 1999;17(9):2639-2648.

2. Demetri GD, et al. Efficacy and safety of imatinib mesylate in advanced gastrointestinal stromal tumors. $N$ Engl J Med. 2002;347(7):472-480.

3. Druker BJ, et al. Effects of a selective inhibitor of the Abl tyrosine kinase on the growth of Bcr-Abl positive cells. Nat Med. 1996;2(5):561-566.

4. Kris MG, et al. Using multiplexed assays of oncogenic drivers in lung cancers to select targeted drugs. JAMA. 2014;311(19):1998-2006.

5. Lopez-Chavez A, et al. Molecular profiling and targeted therapy for advanced thoracic malignancies: a biomarker-derived, mul- 
tiarm, multihistology phase II basket trial. J Clin Oncol. 2015;33(9):1000-1007.

6. Mok TS, et al. Gefitinib or carboplatin-paclitaxel in pulmonary adenocarcinoma. N Engl J Med. 2009;361(10):947-957.

7. Chapman PB, et al. Improved survival with vemurafenib in melanoma with BRAF V600E mutation. $N$ Engl J Med. 2011;364(26):2507-2516.

8. Garraway LA. Genomics-driven oncology: framework for an emerging paradigm. J Clin Oncol. 2013;31(15):1806-1814.

9. Meric-Bernstam F, Farhangfar C, Mendelsohn J, Mills GB. Building a personalized medicine infrastructure at a major cancer center. J Clin Oncol. 2013;31(15):1849-1857.

10. Brannon AR, et al. Comparative sequencing analysis reveals high genomic concordance between matched primary and metastatic colorectal cancer lesions. Genome Biol. 2014;15(8):454.

11. Cheng DT, et al. Memorial Sloan Kettering-Integrated Mutation Profiling of Actionable Cancer Targets (MSK-IMPACT): A Hybridization Capture-Based Next-Generation Sequencing Clinical Assay for Solid Tumor Molecular Oncology. J Mol Diagn. 2015;17(3):251-264.

12. Frampton GM, et al. Development and validation of a clinical cancer genomic profiling test based on massively parallel DNA sequencing. Nat Biotechnol. 2013;31(11):1023-1031.

13. Van Allen EM, et al. Whole-exome sequencing and clinical interpretation of formalin-fixed, paraffin-embedded tumor samples to guide precision cancer medicine. Nat Med. 2014;20(6):682-688.

14. Wagle N, et al. High-throughput detection of actionable genomic alterations in clinical tumor samples by targeted, massively parallel sequencing. Cancer Discov. 2012;2(1):82-93.

15. Won HH, Scott SN, Brannon AR, Shah RH, Berger MF. Detecting somatic genetic alterations in tumor specimens by exon capture and massively parallel sequencing. J Vis Exp. 2013;(80):e50710.

16. Beltran $\mathrm{H}$, et al. Whole-exome sequencing of metastatic cancer and biomarkers of treatment response. JAMA Oncol. 2015;1(4):466-474.

17. Boland GM, et al. Clinical next generation sequencing to identify actionable aberrations in a phase I program. Oncotarget. 2015;6(24):20099-20110.

18. Johnson DB, et al. Enabling a genetically informed approach to cancer medicine: a retrospective evaluation of the impact of comprehensive tumor profiling using a targeted next-generation sequencing panel. Oncologist. 2014;19(6):616-622.

19. Schwaederle M, et al. On the Road to Precision Cancer Medicine: Analysis of Genomic Biomarker Actionability in 439 Patients. Mol Cancer Ther. 2015;14(6):1488-1494.

20. Meric-Bernstam F, et al. Feasibility of Large-Scale Genomic Testing to Facilitate Enrollment Onto Genomically Matched Clinical Trials. J Clin Oncol. 2015;33(25):2753-2762.

21. Jameson GS, et al. A pilot study utilizing multi-omic molecular profiling to find potential targets and select individualized treatments for patients with previously treated metastatic breast cancer. Breast Cancer Res Treat. 2014;147(3):579-588.

22. Gray SW, Hicks-Courant K, Cronin A, Rollins BJ, Weeks JC. Physicians' attitudes about multiplex tumor genomic testing. $J$ Clin Oncol. 2014;32(13):1317-1323.

23. Von Hoff DD, et al. Pilot study using molecular profiling of patients' tumors to find potential targets and select treatments for their refractory cancers. J Clin Oncol. 2010;28(33):4877-4883.

24. Tsimberidou AM, et al. Personalized medicine in a phase I clinical trials program: the MD Anderson Cancer Center initiative. Clin Cancer Res. 2012;18(22):6373-6383.

25. Le Tourneau C, et al. Molecularly targeted therapy based on tumour molecular profiling versus conventional therapy for advanced cancer (SHIVA): a multicentre, open-label, proof-of-concept, randomised, controlled phase 2 trial. Lancet Oncol. 2015;16(13):1324-1334.

26. MacConaill LE, et al. Prospective enterprise-level molecular genotyping of a cohort of cancer patients. J Mol Diagn. 2014;16(6):660-672.

27. Spencer DH, et al. Detection of FLT3 internal tandem duplication in targeted, short-read-length, next-generation sequencing data. J Mol Diagn. 2013;15(1):81-93.

28. Ramkissoon SH, et al. Clinical implementation of integrated whole-genome copy number and mutation profiling for glioblastoma. Neuro-oncology. 2015;17(10):1344-1355.

29. Mandelker D, Dal Cin P, Jacene HA, Armand P, Stone RM, Lindeman NI. Refractory myeloid sarcoma with a FIP1L1-PDGFRA rearrangement detected by clinical high throughput somatic sequencing. Exp Hematol Oncol. 2015;4:30.

30. Doyle LA, et al. Ewing sarcoma mimicking atypical carcinoid tumor: detection of unexpected genomic alterations demonstrates the use of next generation sequencing as a diagnostic tool. Cancer Genet. 2014;207(7-8):335-339.

31. Lawrence MS, et al. Mutational heterogeneity in cancer and the search for new cancer-associated genes. Nature. 2013;499(7457):214-218.

32. Gandhi L, et al. Phase I study of neratinib in combination with temsirolimus in patients with human epidermal growth factor receptor 2-dependent and other solid tumors. J Clin Oncol. 2014;32(2):68-75

33. Yasuda H, et al. Structural, biochemical, and clinical characterization of epidermal growth factor receptor (EGFR) exon 20 insertion mutations in lung cancer. Sci Transl Med. 2013;5(216):216ra177.

34. Jackman D, et al. Clinical definition of acquired resistance to epidermal growth factor receptor tyrosine kinase inhibitors in non small-cell lung cancer. J Clin Oncol. 2010;28(2):357-360.

35. Gao J, et al. Integrative analysis of complex cancer genomics and clinical profiles using the cBioPortal. Sci Signal. 2013;6(269):p11

36. Cerami E, et al. The cBio cancer genomics portal: an open platform for exploring multidimensional cancer genomics data. Cancer Discov. 2012;2(5):401-404.

37. Zhang Z, et al. Activation of the AXL kinase causes resistance to EGFR-targeted therapy in lung cancer. Nat Genet. 2012;44(8):852-860.

38. Rho JK, et al. MET and AXL inhibitor NPS-1034 exerts efficacy against lung cancer cells resistant to EGFR kinase inhibitors because of MET or AXL activation. Cancer Res. 2014;74(1):253-262.

39. Levin PA, Brekken RA, Byers LA, Heymach JV, Gerber DE. Axl Receptor Axis: A New Therapeutic Target in Lung Cancer. $J$ 
Thorac Oncol. 2016;11(8):1357-1362.

40. Drilon A, et al. Broad, hybrid capture-based next-generation sequencing identifies actionable genomic alterations in lung adenocarcinomas otherwise negative for such alterations by other genomic testing approaches. Clin Cancer Res. 2015;21(16):3631-3639.

41. Iyer G, et al. Genome sequencing identifies a basis for everolimus sensitivity. Science. 2012;338(6104):221

42. Wagle N, et al. Activating mTOR mutations in a patient with an extraordinary response on a phase I trial of everolimus and pazopanib. Cancer Discov. 2014;4(5):546-553.

43. Jones S, et al. Personalized genomic analyses for cancer mutation discovery and interpretation. Sci Transl Med. 2015;7(283):283ra53.

44. Garofalo A, et al. The impact of tumor profiling approaches and genomic data strategies for cancer precision medicine. Genome Med. 2016;8(1):79.

45. Seifert BA, et al. Germline analysis from tumor-germline sequencing dyads to identify clinically actionable secondary findings Clin Cancer Res. 2016;22(16):4087-4094.

46. Chau NG, et al. Incorporation of next-generation sequencing into routine clinical care to direct treatment of head and neck squamous cell carcinoma. Clin Cancer Res. 2016;22(12):2939-2949.

47. Harris MH, et al. Multicenter feasibility study of tumor molecular profiling to inform therapeutic decisions in advanced pediatric solid tumors: The Individualized Cancer Therapy (iCat) Study. JAMA Oncol. 2016;2(5):608-615.

48. Mason EF, et al. Detection of activating MAP2K1 mutations in atypical hairy cell leukemia and hairy cell leukemia variant Leuk Lymphoma. 2017;58(1):233-236.

49. Redig AJ, et al. Clinical and molecular characteristics of NF1-mutant lung cancer. Clin Cancer Res. 2016;22(13):3148-3156.

50. Hwang DH, et al. KRAS and NKX2-1 mutations in invasive mucinous adenocarcinoma of the lung. J Thorac Oncol. 2016;11(4):496-503.

51. Awad MM, et al. MET exon 14 mutations in non-small-cell lung cancer are associated with advanced age and stage-dependent MET genomic amplification and c-Met overexpression. J Clin Oncol. 2016;34(7):721-730.

52. Gao X, Sholl LM, Nishino M, Heng JC, Jänne PA, Oxnard GR. Clinical Implications of Variant ALK FISH Rearrangement Patterns. J Thorac Oncol. 2015;10(11):1648-1652.

53. Lee JJ, et al. Targeted next-generation sequencing reveals high frequency of mutations in epigenetic regulators across treatment-naïve patient melanomas. Clin Epigenetics. 2015;7:59.

54. Tanizaki J, et al. Identification of oncogenic and drug-sensitizing mutations in the extracellular domain of FGFR2. Cancer Res. 2015;75(15):3139-3146. 